\title{
Gastrorresistant bio-hybrid beads for indomethacin delivery aiming the oral route
}

\author{
Flávia Monique Rocha Bonetti, Lígia Nunes de Morais Ribeiro and Eneida de Paula
}

\begin{abstract}
Indomethacin is a non-steroidal anti-inflammatory used for many disorders. The oral preferred route causes severe side effects in the gastrointestinal tract, which is exacerbated in chronic diseases, once that a continued administration is necessary. In this work, our goal is the development of a multifunctional solid pharmaceutical form composed of a Nanostructured Lipid Carrier (NLC) prepared with coconut oil further incorporated in (chitosan and xanthan) biopolymers, which combined additional properties to the pharmaceutical form. The resultant bio-hybrids beads were successfully prepared, NLC long-term stability (followed by a year at room temperature), excellent swelling properties and in vitro sustained release profile, for up to 26 hours simulating physiological conditions. These multifaceted bio-hybrid beads seem to be promising for the oral administration of indomethacin in the treatment of chronic inflammatory diseases.
\end{abstract}

\section{Key words:}

Indomethacin, Nanostructured Lipid Carrier, Biopolymers.

\section{Introduction}

Indomethacin (INDO) is a non-steroidal antiinflammatory drug broadly applied for numerous applications, being mainly orally administered. Such route causes several side effects in the gastrointestinal tract, which is intensified in chronic diseases, where prolonged administration is required. Nanostructured lipid carriers are composed of a mixture between solid and liquid lipids at room temperature, and surfactant (1). Additional properties may be achieved through the use of functional excipients (2), as is the case of coconut oil, which has bactericidal, antioxidant and emollient activities. Moreover, the use of biopolymeric materials as matrices can offer additional advantages to the systems, such as mucoadhesion and gastrorresistance, which is extremely important to improve the performance of drug delivery systems (DDS) aiming the oral route. Chitosan is a mucoadhesive biopolymer obtained from crabs, widely used as DDS. The major limitation of chitosan is its sensitivity to acidic mediums, as the gastric fluids. Xanthan is a gum synthesized from Xanthomas and due to high viscosity and high swelling properties; it is considered a natural rheology improver. This work aimed the development of bio-hybrid beads for the indomethacin sustained delivery after oral administration. The lipid component (NLC) is composed of myristyl myristate and coconut oil, while the biopolymeric part is composed of xanthan and chitosan. A blend of INDO-containing NLC formulation and chitosan was processed as beads to provide mucoadhesive properties and spherical shape. Such hybrid beads were then coated by xanthan, in order to protect chitosan from the solubilization effect of the contact with the acidic gastric fluids. Then, if the entire beads reach the intestinal mucosal, chitosan will be able to provide mucoadhesion.

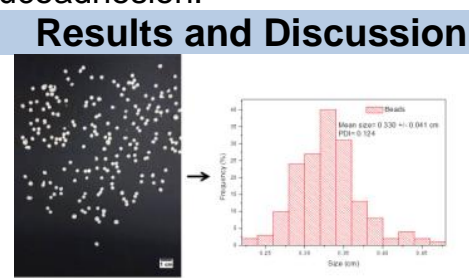

Image 1. Digital photo of the prepared beads and its size dispersion.
A long-term, stable INDO-loaded NLC formulation, compatible with chitosan and xanthan was obtained, providing easily prepared elegant mucoadhesive beads. They showed size around $0.3 \mathrm{~cm}$ with a monodisperse distribution ( $\mathrm{PDI}=0.124)$, calculated by Image J software. In simulated physiologic condition, they showed better swelling properties (tested by the water absorption study) and prolonged INDO in vitro release profile than non-encapsulated INDO-based beads. The xanthan covering sustained the beads delivery of INDO (only $80 \%$ delivered after $26 \mathrm{~h}$ ). In the first $2 \mathrm{~h}$ of experiment (gastric fluids), all the hybrid beads prevented the burst release effect of indomethacin.

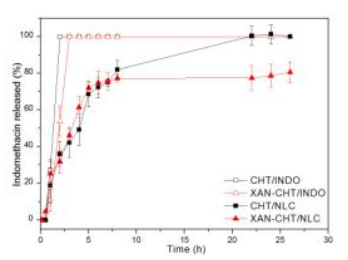

Image 2. Indomethacin in vitro release profile from hybrid (NLC-polymer) and polymer beads, detected by UV-vis at $37^{\circ} \mathrm{C}$. The experiment was conducted at $\mathrm{pH} 1.2$ for $2 \mathrm{~h}$ and 6.8 for $24 \mathrm{~h}$, simulating the oral administration.

\section{Conclusions}

The xanthan-chitosan beads incorporating INDOloaded NLC were successfully prepared and exhibited compatibility between the lipid and biopolymeric components. These hybrid systems presented physical resistance to the disruption in contact with gastric and intestinal simulated fluids, exhibiting a prolonged in vitro release profile for indomethacin. These gastrorresistant beads are promising in the delivery of indomethacin and other similar molecules, for oral administration.

\section{Acknowledgement}

\section{FAPESP (\#14/14457-5, \#14/25372-0)}

${ }^{1}$ Ribeiro LNM, Franz-Montan M, Breitkreitz MC, Alcântara ACS, Castro SR, Guilherme VA, et al. Eur J Pharm Sci. 2016; 93:192-202. ${ }^{2}$ Ribeiro LNM, Breitkreitz MC, Guilherme VA, Rodrigues da Silva GH, Couto VM, Castro SR, et al. Eur J Pharm Sci . 2017; 106: 102-112. 